\title{
SUBOPTIMAL RAKE FINGER ALLOCATION: PERFORMANCE AND COMPLEXITY TRADEOFFS
}

\author{
Konstantinos B. Baltzis — John N. Sahalos *
}

\begin{abstract}
Optimal finger placement improves significantly the performance of RAKE receivers. However, due to its high complexity, it is rarely applied in mobile systems with large channel spread. In this paper, we evaluate the merits of suboptimal finger allocation in terms of performance and complexity. A subset of the RAKE fingers is optimally positioned based on the received signal correlation properties while the rest of them are uniformly distributed within the channel spread. The tradeoffs between performance and complexity of the method are discussed. Results show that optimizing half finger positions lead to similar performance with the full optimization scheme. Finally, comparisons with conventional and optimal receivers exhibit the advantages of the method.
\end{abstract}

K e y w or d s: spread spectrum communication, RAKE receiver, correlation, optimization methods, wideband channel

\section{INTRODUCTION}

In recent year, cellular communications have experienced significant evolution. Third generation $(3 \mathrm{G})$ wireless systems are required to deal with a variety of high data rate applications and promise to offer a vast range and diversity of converged devices, services, and networks, [1-3]. The rapid growth of mobile access and quality of service demands is a major challenge for engineers to propose new methods and techniques that improve system performance. Wideband Code Division Multiple Access (WCDMA) is an effective wireless access technology that supports variable and high data rate services and offers high system capacity, deployed worldwide to provide $3 \mathrm{G}$ mobile systems and services, [4].

Todays subscriber terminals and base stations employ RAKE receivers which collect signal energy that has been dispersed in time by the multipath radio channel. A RAKE receiver consists of fingers which collect the resolvable multipaths. In practice, each finger is an independent receiver which serves to compose and demodulate received signal components. After despreading by a local copy of the delayed version of the transmitter spreading sequence, the signals are suitably combined to perform rake diversity.

An important issue in the design of a RAKE receiver is finger placement. Finger spacing usually equals the chip period, an approach that is not always a good compromise between performance and complexity. In fact, this is the optimal allocation scheme only for the maximal ratio combiner (MRC), [5-6], under the assumption of independent finger signals, [7]. In [8], a combining rule based on maximum likelihood (ML) principles, [5-6], improved system performance by setting finger spacing below the chip duration. Similar principles were applied and evaluated in $[9-18]$, for the estimation of optimum finger settings.
For example, in the Maximum Power Minimum Correlation (MPMC) RAKE receiver, [16], [18], finger placement is determined by the simultaneous maximization of the sum of squares of average received signal power in each finger and minimization of the sum of squares of the cross-correlation between them. In this case, the solution of a multi-objective optimization problem gives the optimal finger settings. In another promising approach ${ }^{1}$, the generalized RAKE (G-RAKE), [12], [15], finger allocation is based on the maximization of the instantaneous signal-to-noise ratio $(S N R)$ averaged over the channel coefficients. A search in a window of potential delays to find the set that optimizes the performance criterion takes place. This window spans from several chip periods before the first multipath component to several chip periods after the latest arriving one.

As WCDMA evolves to higher-bit-rate applications, advanced receiver technology is used to improve coverage and quality of service. However, the increased complexity of structures like the ones mentioned above is a major drawback, especially in wideband channels with large energy and delay spread. Nowadays, a major challenge in electrical and communication engineering is the development of methods and techniques that significantly reduce the computational and hardware complexity of the receivers with a reasonable performance loss (see, for example [20-25]).

Here, we discuss a suboptimal RAKE finger allocation method. In this proposal, only a subset of the fingers is optimally positioned using the MPMC criterion, [16], [18]. Determination of their delays is a multi-objective optimization problem based on the correlation properties of the signal components in each finger. The rest of them

${ }^{1}$ The G-RAKE has been included, [15], from Ericsson for the U350 and U360 platforms for High-Speed Downlink Packet Access (HSDPA) services, [19].

\footnotetext{
* RadioCommunications Laboratory, Section of Applied and Environmental Physics, Department of Physics, Aristotle University of Thessaloniki, Thessaloniki, Greece; kmpal@physics.auth.gr, sahalos@auth.gr
} 


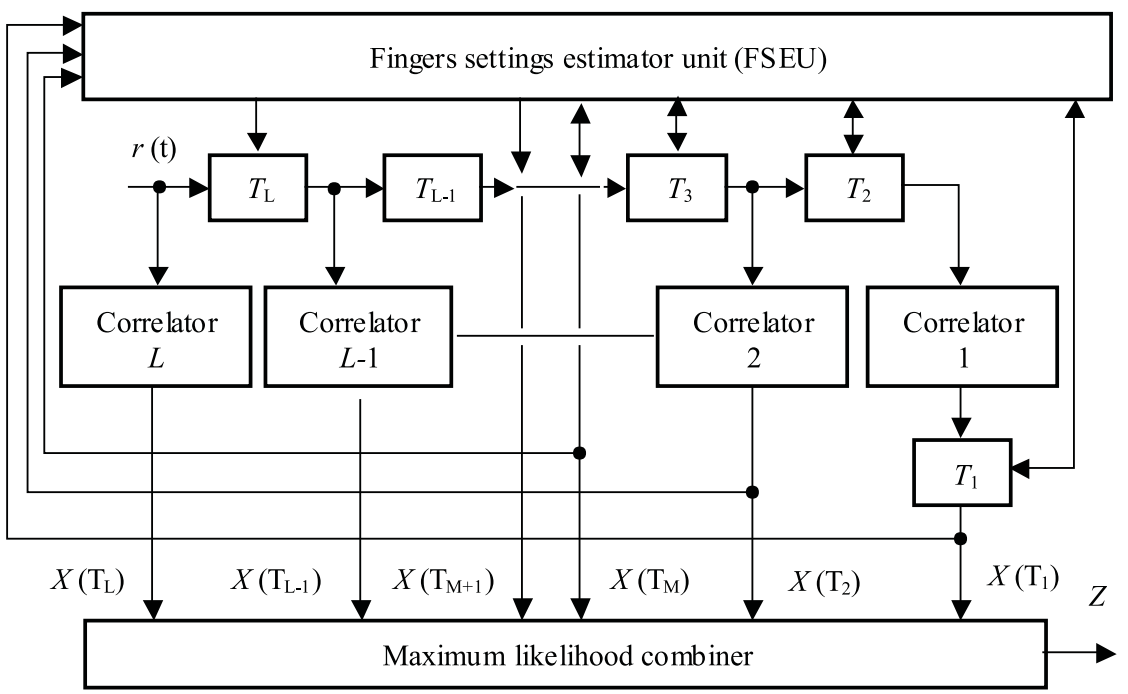

Fig. 1. Receiver model

are uniformly distributed at distances equal to the spacing between the last two optimally positioned ones. Maximum likelihood (ML) principles, [5-6], [8], determine the decision variable. The impact of the number of the optimized fingers is investigated in terms of performance and complexity. Comparisons with relevant methods evaluate the merits of the proposal.

The MPMC RAKE receiver has shown an improved performance compared to other proposals in the published literature. However, its high complexity restricted its use in channels with large energy and delay spread. On the other hand, the S-MPMC RAKE, [25], was far simpler but its poor performance in wideband channels was its major drawback. In this work, the authors investigate the performance and complexity tradeoffs when optimizing (using a modified version of the MPMC criterion) a subset of the total available fingers. The paper aims to the derivation of conclusions that may be helpful for the design and practical implementation of an effective solution for wideband environments.

The remainder of the paper is organized as follows: In Section 2, the transmitter, the channel model and the proposed receiver architecture are described. The modified MPMC criterion is also presented. Section 4 provides results and discussions. Finally, in Section 4 conclusions are drawn.

\section{THEORETICAL PART}

This Section consists of three parts. In the beginning, we describe the transmitter and channel model. Then, we present the proposed receiver architecture. In practice, the proposal extends the MPMC RAKE proposed in [16], [18], considering that only a subset of the total finger positions is optimized. In the rest of the paper, we refer to the proposed receiver as aRC MPMC RAKE (notation $\mathrm{RC}$ stands for reduced complexity; parameter $a$ is the ratio of the optimized fingers $M$ to the total available fingers $L$ ). The Section ends with a brief description of the modified MPMC criterion.

\subsection{Transmitter and channel model}

We consider a direct sequence CDMA communication system with $K$ simultaneous users, the desired user (user $0)$ and $K-1$ interferers. The binary data sequence of each user is modulated by a unique spreading code sequence, such that $N$ continuous chips are modulated by one bit. Therefore, the processing gain $N$ is the ratio of the bit period $T_{b}$ to the chip period $T_{c}$. The user-specific spreading codes are assumed to be mutually orthogonal. For simplicity, we also assume that the signal energy per bit $E_{b}$ is equal for each user. Therefore, the equivalent low-pass BPSK-modulated transmitted signal of the $k^{t h}$ user is

$$
y_{k}(t)=\sqrt{\frac{2 E_{b}}{N}} \sum_{n=-\infty}^{\infty} b_{i n t(n / N)}^{k} a_{n}^{k} h\left(t-n T_{c}\right)
$$

where $\left\{b_{n}^{k}\right\}$ and $\left\{a_{n}^{k}\right\}$ are the binary data and spreading code sequences of the $k^{t h}$ user and $h(t)$ is the normalized transmitted chip waveform.

The radio channel is modeled as a wide-sense stationary uncorrelated scattering (WSSUS) frequency-selective Rayleigh-fading one. The total received signal at the receiver front-end can be written, [26], as

$$
r(t)=\sum_{k=0}^{K-1} \int_{-\infty}^{\infty} \beta_{k}\left(\tau ; t-\tau_{k}\right) y_{k}\left(t-\tau_{k}-\tau\right) d \tau+n(t)
$$

where $n(t)$ is a low-pass equivalent process of Additive White Gaussian Noise (AWGN) with double-sided power spectral density $N_{0} / 2, \tau_{k}$ is the time of arrival of the $k^{t h}$ user signal and $\beta_{k}(\tau ; t)$ is the channel impulse response 
of the $k^{t h}$ user link at delay $\tau$ and time instant $t$. The last is modeled as a complex zero-mean Gaussian random process; its autocorrelation gives the power delay profile (PDP) of the channel, [6].

\subsection{Receiver architecture}

Figure 1 depicts the $M / L$ RC MPMC RAKE receiver model. The receiver consists of $L$ fingers and is matched to the desired user PN spreading code sequence. The received signal $r(t)$ is passed through a tapped delay line (TDL) with fingers positioned at $T_{i}, i=1 \ldots L$. In each finger, the received signal is despread by passing through a correlator matched to the desired user spreading code sequence. Notice that an additional timing offset is introduced through the filter at the first correlator output. In our analysis, we assume perfect knowledge of the chip waveform shaping filters in transmitter and receiver. Also, the receiver knows the exact timing of the desired user signal and the channel impulse response. The last is typically estimated using pilot bits or a pilot channel.

Determination of the fingers settings is performed in the fingers settings estimator unit (FSEU). The unit has knowledge of the first $M$ finger delays and the ability to adjust them; it can also modify the settings of the rest $L-M$ fingers (see, correspondingly, in Fig. 1, the double and the downwards arrows between FSEU and the delay taps). In the FSEU, the autocorrelation of the outputs of the first $M(2 \leq M \leq L)$ fingers $^{2}$ ( $i e$ the average received signal power in each finger) and the cross-correlation between them are calculated. Then the sum of squares of the autocorrelations and the sum of squares of the cross-correlations are calculated. Finally, the modified MPMC criterion, see next subsection, is applied to estimate the suboptimal finger allocation.

The output of each finger is, [8],

$$
X(t)=X_{d}(t)+X_{s}(t)+X_{k}(t)+X_{n}(t)
$$

where $X_{d}(t), X_{s}(t), X_{k}(t)$, and $X_{n}(t)$ are the desired user, the intersymbol interference (ISI), the multi-user interference (MUI), and the AWGN components, respectively. These are

$$
\begin{gathered}
X_{d}(t)=\sqrt{2 E_{b}} b_{1}^{0} \beta_{0}(t) \otimes R_{h h}(t) \\
X_{s}(t)=\sqrt{2 E_{b}} \sum_{n=-\infty, n \neq 0}^{\infty} \beta_{0}(t) \otimes d_{n}^{0} R_{h h}\left(t-n T_{c}\right) \\
X_{k}(t)=\sqrt{2 E_{b}} \sum_{k=1}^{K} \sum_{n=-\infty}^{\infty} \beta(t) \otimes d_{n}^{k} R_{h h}\left(t-n T_{c}-\tau_{k}\right) \\
X_{n}(t)=\frac{1}{\sqrt{N}} \sum_{\lambda=0}^{N-1} n(t) \otimes a_{\lambda}^{0} h\left(-t-\lambda T_{c}\right)
\end{gathered}
$$

where $\otimes$ denotes the convolution operator, $b_{1}^{0}$ is the first bit of the desired user data sequence, $R_{h h}(t)$ is the autocorrelation function of the chip waveform, and $d_{n}^{k}$ is the discrete cross-correlation function between the desired and the $k^{\text {th }}$ user given by

$$
d_{n}^{k}=\frac{1}{N} \sum_{m=0}^{N-1} b_{i n t[(m+n) / N]}^{k} a_{m+n}^{k} a_{m}^{0}
$$

Using (3)-(8), and applying ML principles, [8], the decision variable $Z$ is finally determined at the output of the maximum likelihood combiner.

\subsection{The modified MPMC criterion}

Energy maximization and correlation minimization at the fingers outputs of a RAKE receiver improve its performance. However, it has been found, [16], [18], that optimal performance is obtained when a simultaneous maximization of the sum of squares of average received signal power in each finger and minimization of the sum of squares of autocorrelation between each pair of fingers takes place. This rule is known as Maximum Power Minimum Correlation (MPMC) criterion and it is translated into a multi-objective optimization problem, [27-28].

In the following paragraphs, we present the modified MPMC criterion which is applied in our proposal. In this, finger allocation considers the correlation properties of the first $M$ fingers (in the MPMC criterion, $M$ equals $L)$. Setting $\boldsymbol{T}_{0}=\left[T_{1}, T_{2}, \ldots, T_{M}\right]$, the modified MPMC criterion is defined as

$$
\begin{gathered}
\text { find } \boldsymbol{T}_{0}: \max _{\boldsymbol{T}_{0}} \boldsymbol{F}\left(\boldsymbol{T}_{0}\right) \\
\text { subject to: } T_{i} \in\left[\tau_{i-1}^{0}, \tau_{i}^{0}\right], \quad i=1,2 . . M
\end{gathered}
$$

where $\tau_{0}^{0}=0$ and $\tau_{i}^{0}=g^{-1}[G(0)+(2 i+1) /(2 L+1)]$, $i=1,2 . . M$, the elements of an $M+1$ vector related to the initial fingers settings. Notations $g^{-1}(\cdot)$ and $G(\cdot)$ stand for the inverse function and the antiderivative of the channel PDP, respectively. The objective function is the $\boldsymbol{F}\left(\boldsymbol{T}_{0}\right)=\left\{f_{1}\left(\boldsymbol{T}_{0}\right), f_{2}\left(\boldsymbol{T}_{0}\right)\right\}$ where

$$
f_{1}\left(\boldsymbol{T}_{0}\right)=\sum_{i=1}^{M}\left\{\boldsymbol{E}\left[\left|X\left(T_{i}\right)\right|^{2}\right]\right\}^{2}
$$

the sum of squares of the autocorrelation in each of the first $M$ fingers and

$$
f_{2}\left(\boldsymbol{T}_{0}\right)=\left\{\sum_{i=1}^{M} \sum_{j=i+1}^{M}\left\{\boldsymbol{E}\left[X\left(T_{i}\right) X^{*}\left(T_{j}\right)\right]\right\}^{2}\right\}^{-1}
$$

the inverse of the sum of squares of the cross-correlation between each pair of them.

Constraints of (10) have been derived through an exhaustive search. Our simulations have shown that we should consider $2 L+1$ sequential time intervals with ranges $\Delta \tau_{i}$. The characteristics of these intervals are that integration of the PDP expression over each one gives the same result. After calculating all the $\Delta \tau_{i}$, the rule that determines the search window for each finger setting is

\footnotetext{
${ }^{2}$ The cases $M=L$ and $M=2$ describe the MPMC and the S-MPMC RAKE, respectively.
} 


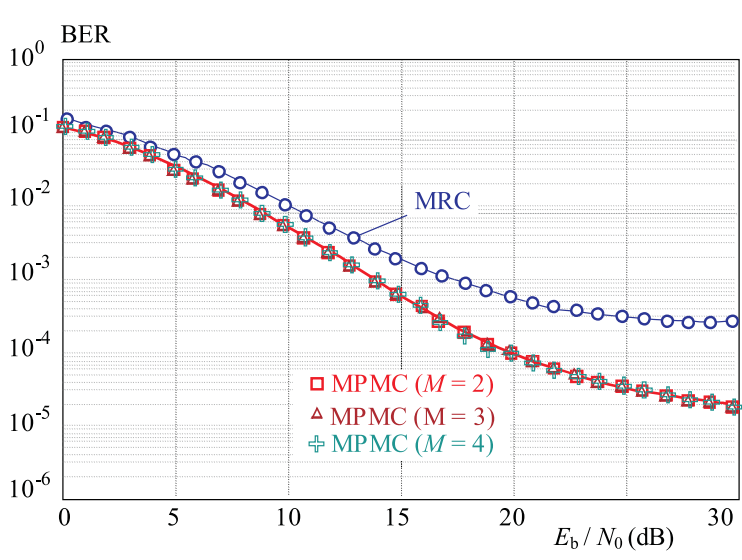

Fig. 2. Bit error rate versus $E_{b} / N_{0},(K=10)$

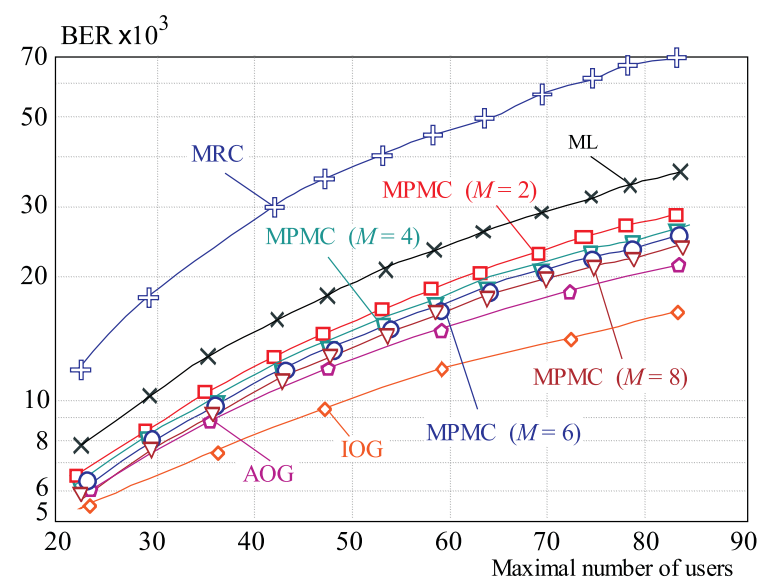

Fig. 4. Comparative performance of 8-finger RAKE receivers, $\left(E_{b} / N_{0}=10 \mathrm{~dB}\right)$

$$
T_{i} \in\left\{\begin{array}{l}
{\left[0, \sum_{j=1}^{3} \Delta \tau_{j}\right], \quad i=1} \\
{\left[\sum_{j=1}^{2 i-1} \Delta \tau_{j}, \sum_{j=1}^{2 i+1} \Delta \tau_{j}\right], \quad i=2,3 . . M}
\end{array}\right.
$$

As an example, in a uniform channel with maximum delay spread $t_{\max }$, (10) becomes

$$
\text { subject to }:\left\{\begin{array}{l}
T_{1} \in\left[0, \frac{3 t_{\max }}{2 L+1}\right] \\
T_{i} \in\left[\frac{(2 i-1) t_{\max }}{2 L+1}, \frac{(2 i+1) t_{\max }}{2 L+1}\right], \\
i=2,3 . . M
\end{array}\right.
$$

After the calculation of $\boldsymbol{T}_{0}$ the positions of the rest of $L-M$ fingers are adjusted. The spacing between each of them is $\tau=T_{M}-T_{M-1}$. Finally, fingers settings are

$$
\begin{array}{r}
\boldsymbol{T}_{o p t}=\left[T_{1}, T_{2} \ldots, T_{M}, T_{M}+\tau, T_{M}+\right. \\
\left.+2 \tau, \ldots, T_{M}+(L-M) \tau\right]
\end{array}
$$

In the simulations, the lexicographic method, [27-29], has been used to solve the optimization problem with the maximization of $f_{1}\left(\boldsymbol{T}_{0}\right)$ to be the premier importance optimization problem. Notice that the method is

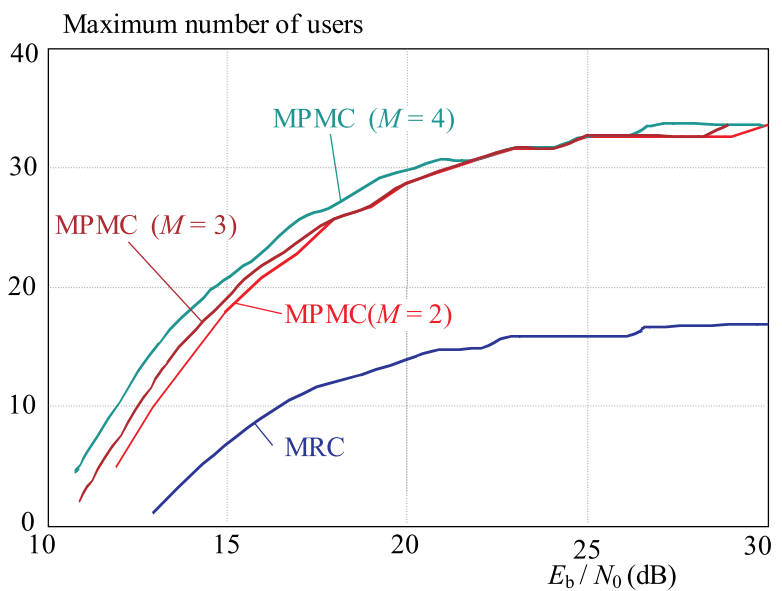

Fig. 3. Maximum number of users versus $E_{b} / N_{0},\left(B E R \leq 10^{-3}\right)$

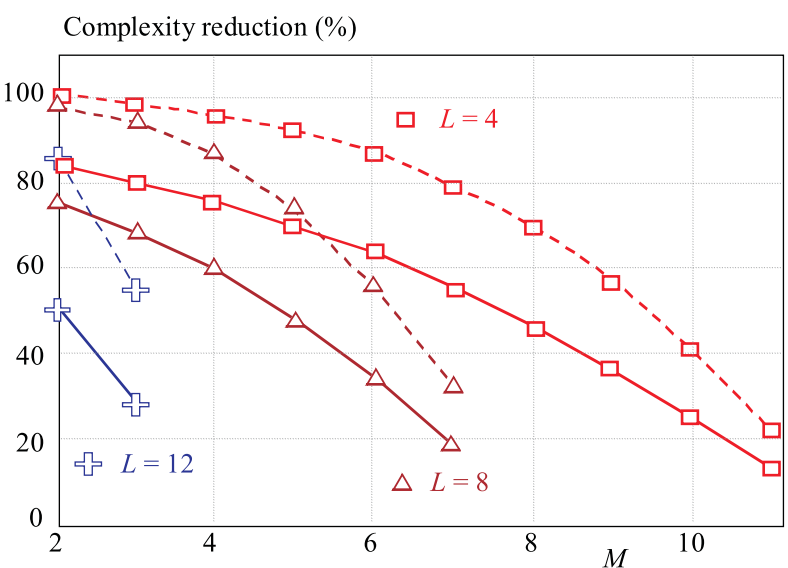

Fig. 5. $H C R$ (solid) and $C C R$ (dotted) for varied $M$ and $L$

not the most appropriate for this kind of problems. For example, particle swarm optimization (PSO) algorithms or a Pareto based methods are preferred in the solution of multi-objective optimization problems of this kind (see, for example, [30-33]). However, its simplicity and low computational cost allows its use in real-time applications. In fact, comparisons with both methods have given similar results in a shorter amount of time. In the Appendix, we further propose a simplified optimization method which leads to similar results when parameter $M$ takes small values; however, as the size of the problem increases the method does not always reach the global optimum solution.

\section{RESULTS AND DISCUSSION}

This Section presents examples which demonstrate the dependence of system performance on the number of the optimized fingers. Comparisons with the MRC RAKE, the ML RAKE with constant finger spacing, [8], (it will be mentioned as conventional $\mathrm{ML}$ ), and the $\mathrm{G}-\mathrm{RAKE}$, are also performed. In the examples provided, the processing gain was 256. Without loss of generality, time-limited rectangular chip pulses and a propagation environment 
with uniform PDP have been considered, assumptions common in the analysis and simulation of CDMA systems (see, for example, [34-37]).

First, we examine the case of four-finger RAKE receivers in a uniform channel with maximum delay spread $t_{\max }=2 T_{c}$. For reference reasons, characteristics and performance of the conventional MRC RAKE are also illustrated. In Tab. 1, fingers settings are presented. Notice the small differences when optimizing a subset of the total available fingers, as a result of the small channel delay spread.

Table 1. Finger allocation (in chip perionds) in four-finger RAKE receivers

\begin{tabular}{lccccc}
\hline & & $T_{1}$ & $T_{2}$ & $T_{3}$ & $T_{4}$ \\
\hline MRC & & 0 & 1 & 2 & 3 \\
& $\mathrm{M}=2$ & 0.25 & 0.75 & 1.25 & 1.75 \\
\multirow{2}{*}{$\mathrm{RC}$} & $\mathrm{M}=3$ & 0.21 & 0.74 & 1.27 & 1.80 \\
MPMC & & & & & \\
& $\mathrm{M}=4$ & 0.28 & 0.65 & 1.30 & 1.70 \\
\hline
\end{tabular}

In Figs. 2 and 3, performance characteristics of the receivers in Tab. 1 are illustrated. Fig. 2 shows the bit error rate $(B E R)$ versus $E_{b} / N_{0}$. In Fig. 3 , the maximum number of users allowed in the system in order to have an error probability smaller than 10-3 (a typical value for voice transmission, [38]), versus $E_{b} / N_{0}$ is depicted. Obviously, the performance of the MRC is significantly worst compared to the other implementations. Comparisons between the MPMC structures show that increase in receiver complexity does not practically affect its performance. Practically, in a channel with a small energy and delay spread, optimization of two fingers and allocation of the rest at equal distances with the distances of the first two gives an adequate performance and decreases system complexity.

Our previous analysis has shown that, in a narrowband channel environment, optimization of more than two fingers increases system complexity without performance improvement. In Fig. 4, we study the performance of the $a \mathrm{RC}$ MPMC, MRC, the conventional ML with finger spacing equal to 0.7 chip periods (this value was used in [8] to demonstrate its performance), and the instantaneous optimum (IOG) and average optimum (AOG) $\mathrm{G}-\mathrm{RAKE}^{3}$ in wideband environment. Receivers consist of eight fingers. The propagation channel is the modified wideband ITU vehicular channel model given in [12], [39], with 8 rays of delays $\left\{0, T_{c}, \ldots, 7 T_{c}\right\}$ and average powers $\{0,-2.4,-6.5,-9.4,-12.7,-13.3,-15.4,-25.4\}$ (in dB).

The structures with optimized finger placement show an improved performance compared to the conventional MRC and the conventional ML RAKE. The G-RAKE shows an improved performance comparing to the MPMC RAKE structures. This was expected because in $\mathrm{G}-$ RAKE both fingers settings and weights are optimized. However, in this receiver finger position optimization uses arithmetic methods increasing computational complexity, a serious drawback in a real-time application. From Fig. 4 , we may draw interesting conclusions about the impact of the number of optimized taps on the performance of a RAKE receiver in wideband channels. We notice that optimization of four out of eight fingers gives results close to the optimum. Adding further complexity (6/8RC MPMC RAKE), we obtain a performance level similar to the MPMC RAKE.

A detailed analysis of performance degradation due to the decrease in system complexity is given in Tab. 2 . There, capacity reduction in terms of maximum number of users is presented for structures with different number of optimized fingers at various maximum accepted values of error probability. From Fig. 4 and Tab. 2, we conclude that optimization of half of the fingers lead to a performance almost similar to the full optimized MPMC RAKE.

Table 2. Capacity reduction of 8-finger MPMC RAKE receivers, $\left(E_{b} / N_{0}=10 \mathrm{~dB}\right)$

\begin{tabular}{cccc}
\hline$B E R \times 10^{3}$ & $M=4$ & $M-2$ & $M=6$ \\
& $\%$ & $\%$ & $\%$ \\
\hline 5 & 4.8 & 4.8 & 0 \\
10 & 7.5 & 2.5 & 2.5 \\
15 & 8.8 & 5.3 & 3.5 \\
20 & 8.5 & 4.2 & 1.4 \\
25 & 10.3 & 5.7 & 3.4 \\
\hline
\end{tabular}

Table 3. Number of correlators

\begin{tabular}{ccc}
\hline $\mathrm{L}$ & $\mathrm{M}$ & num_of_corr \\
4 & 2 & 7 \\
4 & 3 & 10 \\
4 & 4 & 14 \\
8 & 2 & 11 \\
8 & 4 & 18 \\
8 & 6 & 29 \\
8 & 8 & 44 \\
\hline
\end{tabular}

Finally, an approximate estimation of the reduction in hardware and computational complexity when optimizing $2 \leq M<L$ instead of $L$ fingers is performed. We examine the hardware complexity reduction $(H C R)$ in terms of the number of correlators. The $\mathrm{L}-$ finger MPMC RAKE has $L^{2} / 2+3 L / 2$ correlators, $\mathrm{L}$ at the outputs of its fingers and $L(L+1) / 2$ in the fingers settings estimator unit. Similarly, the $M / L \mathrm{RC}$ MPMC RAKE has $M(M+1) / 2+L$ correlators. In Tab. 3, the number of correlators (num_of_corr) of the receivers which are

\footnotetext{
${ }^{3}$ The IOG-RAKE finds the optimum finger positions and weights for each realization of channel response; the AOG-RAKE finds a set of fixed positions that minimizes the error probability, [12].
} 
studied in the previous examples is given. It can easily be shown that the $H C R$ as a function of $M$ and $L$ is

$$
H C R=1-\frac{M^{2}+M+2 L}{L(L+3)}
$$

ie it depends on $M^{2}$ for a given $L$.

A similar analysis, about the computational complexity reduction $(C C R)$ can also been made. Here, we study $C C R$ in terms of the number of correlation estimations in the fingers settings estimator unit and the comparisons during the application of the MPMC criterion. Obviously, the complexity reduction is proportional to the number of correlation estimations. The number of comparisons during the application of the MPMC criterion also depends on $M$ and varies according to the optimization algorithm that is used. As an example, for the algorithm presented in the appendix this relation is approximately linear. In this case, we can approximate $C C R$ as a function of $M$ and $L$ as

$$
C C R \approx 1-\frac{M^{2}(M+1)}{L^{2}(L+1)}
$$

$i e$, it depends on $M^{3}$ for a given $L$.

The hardware and approximate computational complexity reduction curves are illustrated in Fig. 5. Notice that the complexity reduction is greater than the hardware reduction. As it has already been mentioned, performance degradation is not significant when half of the finger settings are optimized. In this case, $C C R$ approaches $90 \%$ and $H C R$ is around $60 \%$. However, as long as specific parameters have been considered only, we expect that the total reduction in hardware and computational complexity is smaller in a real system.

In practice, the reduction in computational cost and hardware complexity compensates for the slightly worst performance of the proposal compared to the MPMC RAKE. In any case, parameters such as channel spread, implementation cost, and power consumption must be considered when choosing the number of fingers to be optimized in an RC MPMC RAKE implementation. Definitely, optimization of all the fingers is not the most appropriate solution, especially when a great number of fingers are used (in a wideband channel for example). Optimization of a subset of the total number of RAKE fingers decreases significantly receiver complexity. This reduction compensates for the slightly worst system performance. As it has already been mentioned, optimization of half of the fingers provides a good tradeoff between performance and complexity.

Finally, it has to be mentioned that we have also examined the case where finger allocation was based on the correlation properties of all the fingers but the receiver optimized the positions of only the first $M$. In this case, only the computational cost reduced. However, simulations have shown negligible differences in the results with the approach presented in this paper.

\section{CONCLUSION}

In this paper, we have studied the impact of the optimization of a subset of a RAKE receiver finger settings in terms of performance and complexity. The derived results have shown that the significant reduction in hardware and computational complexity compensates for the decreased performance of the receiver. Simulations have shown that optimization of half of the receiver finger settings gives adequate results. The proposal is an interesting one, especially in wideband channels with large energy and delay spread where a significant number of fingers are needed.

\section{Appendix}

In this Appendix, we propose a simplified optimization method that can be applied for the solution of the MPMC criterion. In practice, the method translates the problem from a single multivariable to $M$ single-variable ones. A major drawback of the method is that it does not guarantee the derivation of a globally optimum solution, especially when the size of the problem increases significantly. However, according to our simulations the method is efficient for small values of $M$. In Tab. A.1 the main steps of the algorithm are presented.

Tab A.1 Main steps of the proposed optimization algorithm

1: Set the initial settings of the fingers to be optimized $\tau_{i}^{0}, i=1,2 \ldots, M$

2: Apply (9) and (10) for the 1st finger considering the rest of them fixed at $\tau_{i}^{0}, i=2,3 \ldots, M$

3: Apply (9) and (10) for the 2nd finger considering the first fixed at the value calculated in steps 2 , 3 , and the rest of them at $\tau_{i}^{0}, i=3,4 \ldots, M$

M+1 Apply (9) and (10) for the $M^{\text {th }}$ finger considering rest fixed at the values calculated in steps $2,3, \ldots$, $\mathrm{M}$

$\mathrm{M}+2$ Set the positions of the rest $L-M$ fingers at distances equal to $T_{M}-T_{M-1}$

\section{REFERENCES}

[1] LI, X.-WU, D.: Power control and channel allocation for real-time applications in cellular networks, Wireless Communications and Mobile Computing 8 No. 6 (2007), 705-713.

[2] MATIASKO, K.-ZABOVSKY, M.: A data replication for mobile environment, Journal of Electrical Engineering 59 No. 5 (2008), 277-280.

[3] RUPP, M.: Video and Multimedia Transmissions over Cellular Networks Analysis, Modeling and Optimization in Live 3G Mobile Networks, John Wiley \& Sons Ltd, 2009.

[4] CHEN, H.-H. : The Next Generation CDMA Technologies, John Wiley \& Sons Ltd, 2007.

[5] LEE, J. S.-MILLER, L. E.: CDMA Systems Engineering Handbook, Artech House, 1998. 
[6] PROAKIS, J. G.-SALEHI, M. : Digital Communications, 5-th Edition, McGraw-Hill, 2007.

[7] DONG, X.-BEAUliEU, N. C. : Optimal maximal ratio-combining with correlated diversity, IEEE Communications Lett 6 No. 1 (2002), 22-24.

[8] KIM, K. J.-KWON, S. Y.-HONG, E. K.-WHANG, K. C. : Effect of tap spacing on the performance of direct-sequence spread spectrum RAKE receiver, IEEE Trans. on Communications 48 6 No. 1 (2000), 1029-1036.

[9] BEJJANI, E-BOUQIER, J.-F-DECACQUERAY, B. : Adaptive channel delays selection for WCDMA mobile system, Proc. IEEE VTS 50th Vehicular Technology Conference (VTC '99), 1 Fall (1999), 203-207.

[10] BALACHANDRAN, K.-CHANG, K. K.-REGE, K. M. : RAKE receiver finger assignment in CDMA terminals with fractionally spaced multipaths, Proc. IEEE VTS 50th Vehicular Technology Conference (VTC '99), 1 Fall (1999), 476-481.

[11] VEJLGAARD, B. N.-MOGENSEN, P.-KNUDSEN, J. B. : Grouped RAKE finger management principle for wideband CDMA, Proc. IEEE VTS 51st Vehicular Technology Conference (VTC '00), 1 (2000), 87-91.

[12] BotTomley, G. E.-OTtOSOn, T.-WANG, Y.-P. E. : A generalized RAKE receiver for interference suppression, IEEE J. of Selected Areas in Communications 18 No. 8 (2000), 1536-1545.

[13] LAU, W. C.-AlOUINI, M.-SIMON, M. K. : Optimum spreadng bandwidth for selective RAKE reception over Rayleigh fading channels, IEEE J. of Selected Areas in Communications 19 No. 6 (2001), 1080-1089.

[14] LI, C.-M.-LI, H.-J.: A novel RAKE receiver finger number decision rule, IEEE Antennas Wireless Propagation Lett 12 No. 6 (2003), 277-280.

[15] BOtTomley, G. E.-CAIRns, D. A.-COZZO, C.-FULGUHM, T. L.-KHAYRALLAH, A. S.-INDELL, P.-SUNDELIN, M.-WANG, Y.-P. E. : Advanced receivers for WCDMA terminal platforms and base stations, Ericsson Review, 2 (2006), 54-58.

[16] BALTZIS, K. B.-SAHALOS, J. N. : On the design of RAKE receivers with non-uniform finger spacing, Proc. 5th International Symposium on Communication Systems, Networks and Digital Signal Processing (CSNDSP '06) (2006), 486-490.

[17] SUI, H.-MASRY, E.-RAO, B. D.: Chip-level DS-CDMA downlink interference suppression with optimized finger placement, IEEE Trans. on Signal Processing 54 No. 10 (2006), 3908-3921.

[18] BALTZIS, K. B.-SAHALOS, J. N. : A novel RAKE receiver design for wideband communications, Wireless Personal Communications 43 No. 4 (2007), 1603-1624.

[19] DAHLMAN, E.-PARKVALL, S.-SKOLD, J.-BEMING, P. : 3G Evolution: HSPA and LTE for place Mobile Broadband, 2nd Edition, Elsevier Ltd, 2008.

[20] KRISHNA, K. M.-MITRA, A.-ARDIL, C. : A simplified single correlator rake receiver for CDMA communications, Proc. of World Academy of Science, Engineering and Technology $\mathbf{8}$ (2005), 106-109.

[21] GEZICI, S.-CHIANG, M.-POOR, H. V.-KOBAYASHI, H.: Optimal and suboptimal finger selection algorithms for MMSE RAKE receivers in impulse radio ultra-wideband systems, EURASIP J. on Wireless Communications and Networking (2006), doi: 10.1155/WCN/2006/84249.

[22] KIM, B. S.-BAE, J.-SONG, I.-KIM, S. Y.-KWON, H. : Optimum and suboptimum rake receivers in impulsive UWB environment, IEEE Trans. on Vehicular Technology 55 No. 6 (2006), 1797-1804.

[23] CASSIOLI, D.-WIN, M. Z.-VALATARO, F.-MOLISCH, A. F.: Low complexity rake receivers in ultra-wideband channels, IEEE Trans. on Wireless Communications 6 No. 4 (2007), $1265-1275$.
[24] JIN, H.-SALEHI, M.: A new finger placement for the generalized RAKE receiver, Proc. 42-nd Annual Conference on Information Sciences and Sysems (CISS '08) (2008), 335-340.

[25] BALTZIS, K. B.: An efficient finger allocation method for the maximum likelihood RAKE receiver, Radioengineering 17 No. 4 (2008), 45-50.

26] JERUCHIM, M. C.-BALABAN, P.-SHANMUGAN, K. S. : Simulation of communications systems: modeling, methodology and techniques, 2nd Edition, Kluwer Academic/Plenum Publishers, 20000.

[27] STADLER, W. : Multicriteria Optimization in Engineering and in the Sciences, Plenum Press, 1988.

[28] ZLOBEC, S.: Stable Parametric Programmings, Kluwer Academic Publishers, 2001.

[29] EHRGOTT, M. : Multicriteria Optimization, Springer, 2005.

[30] BEN-TAL, A.: Characterization of Pareto and lexicographic optimal solutions, Proc. 3rd Conference on Multiple Criteria Decision Making Theory and Application, Springer Verlang, 1979, $1-11$.

[31] GOLDBERG, D. E. : Genetic Algorithms in Search, Optimization and Machine Learning, Addison-Wesley, 1989.

32] COEllo, C. A. C.-LAMONT, G. B.-VANVELDHUIZEN, D. A.: Evolutionary Algorithms for Solving Multi-Objective Problems, 2-nd Edition, Springer, 2007.

33] AWADAllah, M. A.-BAYOUMI, E. H. E.-SOLIMAN, H. M.: Adaptive deadbeat controllers for brushless drives using PSO and ANFIS techniques, Journal of Electrical Engineering 60 No. 1 (2009), 3-11.

34] ABRARDO, A. : Noncoherent MLSE detection of M-DPSK for DS-CDMA wireless system, IEEE Trans. on Vehicular Technology 50 No. 4 (2001), 900-909.

35] KIM, J.-KIM, I.-RO, S.-HOND, D.: Effects of multipath diversity on adaptive QAM in frequency selective Rayleigh fading channels, IEEE Communications Lett. 6 No. 9 (2002), 364-366.

36] HAMILA, R.-LOHAN, E. S.-RENFORS, M. : Subchip multipath delay estimation for downlink WCDMA system based on Teager-Kaiser operator, IEEE Communications Lett. 7 No. 1 (2003), 1-3.

37] ABRARDO, A. : Noncoherent MLSE in DS-CDMA wireless systems with antenna arrays, IEEE Trans. on Vehicular Technology 52 No. 6 (2003), 1435-1446.

[38] GOLSMITH, A.: Wireless Communications, Cambridge University Press, 2005.

[38] 3RD GENERATION PARTHENRSHIP PROJECT, TSG RAN WG4 UE Radio Transmission and Reception (FDD), version 2.0, 1999 .

Received 27 May 2009

Konstantinos B. Baltzis was born in Thessaloniki, Greece in 1973. He received his BSc degree in Physics in 1996, his MSc degree in Electronics and Communications in 1999, and his $\mathrm{PhD}$ degree in Communication Engineering in 2005, all from the Aristotle University of Thessaloniki (AUTh), Greece. He currently works as a research assistant in the RadioCommunications Laboratory of AUTh. He is also a (visiting) assistant professor in the Department of Automation at Alexander Technological Educational Institution of Thessaloniki and a teaching staff member in the Program of Postgraduate Studies in Electronic Physics at AUTh. His current research interests include wideband communications, wireless networks, antennas, microwave systems and optimization methods.

John N. Sahalos biography not available. 